\title{
A Novel Disease-Causing ASPA Gene Mutation (c.432+1 G>C) in an Iranian Patient with Canavan Disease: A Case Report*
}

\author{
Mostafa Neissi ${ }^{1,2}$; Motahareh Sheikh-Hosseini ${ }^{3}$; Javad Mohammadi-Asl ${ }^{4}$ \\ ${ }^{1}$ Department of Genetics, Khuzestan Science and Research Branch, Islamic Azad University, Ahvaz, Iran \\ ${ }^{2}$ Department of Genetics, Ahvaz Branch, Islamic Azad University, Ahvaz, Iran \\ ${ }^{3}$ Department of Medical Genetics, Institute of Medical Biotechnology, National Institute of Genetic \\ Engineering and Biotechnology (NIGEB), Tehran, Iran \\ ${ }^{4}$ Department of Medical Genetics, School of Medicine, Ahvaz Jundishapur University of Medical Sciences, \\ Ahvaz, Iran
}

\begin{abstract}
Canavan disease is an autosomal recessive genetic disease and rare fatal childhood neurological disorder caused by mutations in the ASPA gene, which resulted in a catalytic deficiency of the ASPA enzyme that catalyzes the hydrolysis of N-acetylaspartic acid into aspartate and acetate. Herein, we report an Iranian patient diagnosed with Canavan disease with a novel splice-site mutation in the ASPA gene (NM_000049.4; c.432+1 G>C). This report is based on a homozygous c.432+1 G>C mutation in the $A S P A$ gene identified from an Iranian patient. As a result, a novel homozygous pathogenic mutation on $A S P A$ is the cause of disease in the patient.(International Journal of Biomedicine. 2021;11(4):594-597.)
\end{abstract}

Key Words: Canavan disease $\bullet$ novel mutation $\bullet$ ASPA gene $\bullet$ aspartoacylase $\bullet$ N-acetylaspartic acid

For citation: Neissi M, Sheikh-Hosseini M, Mohammadi-Asl J. A Novel Disease-Causing ASPA Gene Mutation (c.432+1 $\mathrm{G}>\mathrm{C}$ ) in an Iranian Patient with Canavan Disease: A Case Report. International Journal of Biomedicine. 2021;11(4):594-597. doi:10.21103/Article11(4)_CR2

*Published without substantive editing per OFAC guidance.

\section{Abbreviations}

NAA, N-acetylaspartic acid; MRS, magnetic resonance spectroscopy; MRI, magnetic resonance imaging; gDNA, genomic DNA; WES, whole-exome sequencing; ACMG, American College of Medical Genetics and Genomics.

\section{Introduction}

Canavan disease is an autosomal-recessive leukodystrophy and fatal neurological disease which is characterized by developmental delay, neurologic deterioration with severe intellectual disability, and early death. ${ }^{(1)}$ The underlying cause of this disease is the deficiency in the enzyme aspartoacylase, which leads to high levels of $\mathrm{N}$-acetylaspartic acid (NAA) in

Corresponding author: Mostafa Neissi. Department of Genetics, Khuzestan Science and Research Branch, Islamic Azad University, Ahvaz, Iran. E-mail: iammostafaneissi@gmail.com the urine, brain, and body fluids. The $A S P A$ gene mutations are responsible for this deficiency (RefSeq NM_000049.4). ${ }^{(2)}$ ASPA is a catabolic enzyme that is primarily in oligodendrocytes in the central nervous system. ${ }^{(3)}$ ASPA catalyzes the hydrolysis of NAA to generate aspartate and acetate, it is a homodimer and essential in the synthesis of myelin. Patients who are deficient in the ASPA enzyme activity have an abnormal elevation of NAA in the brain. This can be identified by applying magnetic resonance spectroscopy (MRS) even before increasing its concentration in the urine, which is suitable for the early diagnosis of Canavan disease. ${ }^{(4)}$ Clinical symptoms are not manifested at the time of birth; however, the clinical triad of hypotonia, macrocephaly, and head lag often in association with macrocephaly and 
seizures are initial diagnostic manifestations for Canavan disease in early childhood. ${ }^{(5)}$ Neuroimaging presents brain white matter signal abnormalities, and, at later time-points, ventricular enlargement. ${ }^{(6,7)}$ Patients with Canavan disease in the first months of life have dysmyelination, intramyelinic edema, and characteristic spongiform degeneration of the white matter of the brain with impairment of psychomotor development, which is specified by cognitive delay, ataxia, and irritability. In atypical cases, disease onset is postponed until some years after birth when some aspartoacylase enzymatic activity remains. ${ }^{(8,9)}$ Other symptoms involve difficulties in sucking and visual tracking, progressive macrocephaly, and preserved social interactions. Disease progression is marked by atrophy of the optic nerve, spastic tetraparesis, intellectual disability, seizures, and early death. Magnetic resonance imaging (MRI) is routinely used for the diagnosis of the characteristic spongy degeneration of the white matter, which typically shows signal abnormalities of the white matter and the basal ganglia. Up to now, the Human Gene Mutation Database has presented 83 mutations in the ASPA gene (www.hgmd.cf.ac.uk). Novel mutations in some genes can be inherited in an autosomal dominant or autosomal recessive inheritance. ${ }^{(10,11)}$ Herein, we present a novel mutation in the ASPA gene in one patient in an Iranian family with severe Canavan disease.

\section{Case presentation}

Our patient was the second born male child (Fig. 1A; IV:2) of consanguineous parents with no family history of any genetic condition or mental retardation. The parents have two children. The father and mother are first cousins and showed no signs or symptoms of Canavan disease. The 6-year-old girl is healthy and the second child is a boy with 2 years old and has symptoms of Canavan disease. The mother started to notice a delay in acquiring developmental milestones by the age of 4 months as he has the symptoms of hypotonia. At 19 months of age, he had severe developmental delay, a lack of neck support, frontal bossing, and macrocephaly. MRI of the brain revealed a diffuse lesion of the white matter affecting the U-fibers. The diagnosis of Canavan disease was confirmed by the findings of a very high concentration of NAA in urine. The parents of the patient provided written informed consent in accordance with the Helsinki Declaration.

A salting-out method for genomic DNA (gDNA) extraction was done. gDNA sample from peripheral blood lymphocyte of the patient was examined by whole-exome sequencing (WES) technique (Macrogen, Seoul, South Korea) to identify the mutations associated with this disease. A novel homozygous ASPA c.432+1 G>C mutation in exon2/intron2 boundary region (NM_000049.4) was found. Finally, to confirm the presence of the $\overline{A S P A}$ variant, a direct Sanger sequencing method for the patient and his parents were done. So, the specific sets of primers were designed to amplify the mutated sites in the genome by the PCR method. After amplification of $A S P A$ sequences, we sequenced the PCR products directly on the automated genetic analyzer (ABI 3130XL; USA) and the results are represented in Figure 1B. This finding has not been reported in the other Canavan patients.
Furthermore, the splice-site mutation $(\mathrm{c} .432+1 \mathrm{G}>\mathrm{C})$ was classified as pathogenic according to the American College of Medical Genetics and Genomics (ACMG) guidelines. MutationTaster (bioinformatics program) predicted that this mutation is disease-causing.

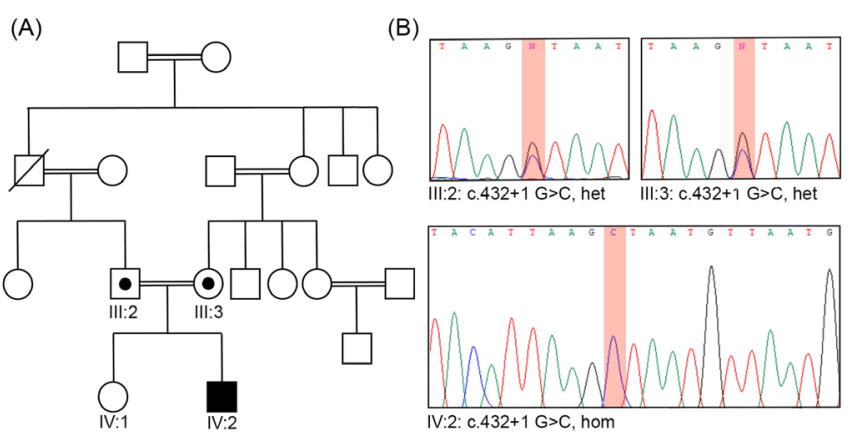

Fig 1. (A) Family pedigree of the patient. The black square represents the patient. Parents have a family history of consanguinity. The unfilled square represents a male, and the circle represents a female. The diagonal line on the square represents a deceased male. The circle in the center of symbols represents heterozygosity. (B) Sequence analysis of the patient and parents revealed that in terms of inheritance, the patient's parents were heterozygous (III:2 and III:3) and the patient (IV:2) was homozygous. The chromatogram shows the splicing mutation, $G$ to $C$ change in the splice donor of intron $2(c .432+1 G>C)$.

\section{Discussion}

Known as Canavan disease, it is an autosomal recessive form of human spongiform leukodystrophy caused by an inborn error of the ASPA activity. ${ }^{(12)}$ The substrate of aspartoacylase enzyme is NAA, which is exclusively synthesized in the brain. NAA is hydrolyzed by aspartoacylase to acetate which is necessary for myelin synthesis and aspartate. ${ }^{(13,14)}$ Aspartoacylase deficiency leads to accumulation of NAA in the brain, causing pathological spongy degeneration of the white matter. ${ }^{(14,15)}$ Clinically, two types of Canavan disease have been reported. The most common type of Canavan disease is the neonatal or infantile form that is more severe in clinical presentation in comparison with the juvenile type of the disease. ${ }^{(16,17)}$ Clinical symptoms of neonatal or infantile Canavan disease commonly begin between the age of 2 to 6 months and appear with lack of neck holding in pull to sit maneuver, axial hypotonia, lethargy, spasticity as well as macrocephaly, poor feeding, developmental regression, and progressive hyperreflexia. Cortical blindness and optic atrophy are followed by seizures in later stages. ${ }^{(17)}$ The clinical course of our patient was consistent with the infantile type of Canavan disease.

Extraction of human cDNA and the ASPA gene helps to explain the molecular basis of Canavan disease. ASPA is the only gene for Canavan disease that is located on chromosome 17p13.2. ${ }^{(16)}$ The examination of mutations in patients with Canavan disease has revealed missense, nonsense, splice-Site, and frameshift mutations, deletions, or insertions. ${ }^{(18)}$ Canavan disease is more prevalent in people of Ashkenazi Jewish than another ethnicity. ${ }^{(19)}$

Several mutations have been reported to have phenotypes associated with their genotypes, such as $698 \mathrm{insC}, \mathrm{X} 314 \mathrm{~W}$, 
P181L, 244delAT, 923delT, C152W, V14G, D249V, and E214X with a severe phenotype. A stop codon or frameshift occurs in many of these mutations that is related to the onset during the first few months after birth ( 2 or 3 months of age). In Jewish populations, E285A and Y231X mutations are correlated with a severe phenotype as well. ${ }^{(20)}$ The mutation that was found in our patient also cause a severe phenotype.

Deficiency of the $A S P A$ activity caused by the nonsense tyr231ter, the missense ala305glu mutation, or the glu285ala mutation establishes that the three coding-sequence mutations are the cause of Canavan disease. ${ }^{(21)}$ The $433-2$ A to $G$ transition in intron 2 (in the splice acceptor site) would result in skipping of exon 3. Additionally, skipping of 94-base exon 3 , in the final transcript will change the reading frame. A frameshift accompanied by an exon-skipping would result in the aspartoacylase deficiency. ${ }^{(22)}$

In 2012, Durmaz et al. reported a novel heterozygous mutation Y88X ( $\mathrm{T}$ to A nucleotide change at codon 88 in exon 2) within the aspartoacylase gene in a consanguineous family with an affected child diagnosed as Canavan disease. This mutation converts the codon for tyrosine (TAT) into a premature termination codon (TAA). ${ }^{(20)}$ Also, in 2015, Ashrafi et al. indicated a novel homozygous missense mutation (c. $202 \mathrm{G}>\mathrm{A}$ ) in the ASPA gene in exon 1 which was found in an Iranian patient. ${ }^{(23)}$ In our case, we presented a novel homozygous pathogenic mutation in ASPA gene (c.432+1 $\mathrm{G}>\mathrm{C}$ ) related to Canavan disease. This mutation was at the 5, splice-site beginning intron 2 , which can cause mis-splicing and alter the reading frame, and consequently, it will probably result in a serious alteration in ASPA protein conformation and leads to the Canavan phenotype. This type of mutation has not been reported in other populations.

\section{Conclusion}

In the present study, we report a 2-year-old Iranian boy with severe Canavan disease who harbors a novel pathogenic homozygous mutation (c.432+1 $\mathrm{G}>\mathrm{C})$ in the $A S P A$ gene. Homozygous mutation as in the intron 2 of $A S P A$ gene in the present case is a novel splice-site mutation that was not reported elsewhere. The mutation that leads to the Canavan disease has been defined in the family; it would make prenatal diagnosis possible and suggest parents with such disorder plan for the next pregnancy.

\section{Acknowledgments}

The authors would like to express their deepest gratitude to the family members for their participation in this study.

\section{Competing Interests} interests.

The authors declare that they have no competing

\section{Disclaimers}

The views expressed in this article are the author's own and do not reflect the official position of the institutions.

\section{References}

1. Pleasure D, Guo F, Chechneva O, Bannerman $\mathrm{P}$, McDonough J, Burns T, Wang Y, Hull V. Pathophysiology and Treatment of Canavan Disease. Neurochem Res. 2020 Mar;45(3):561-565. doi: 10.1007/s11064-018-2693-6.

2. Zaki OK, Krishnamoorthy N, El Abd HS, Harche SA, Mattar RA, Al Disi RS, Nofal MY, El Bekay R, Ahmed KA, George Priya Doss C, Zayed H. Two patients with Canavan disease and structural modeling of a novel mutation. Metab Brain Dis. 2017 Feb;32(1):171-177. doi: 10.1007/s11011016-9896-9.

3. Madhavarao CN, Moffett JR, Moore RA, Viola RE, Namboodiri MA, Jacobowitz DM. Immunohistochemical localization of aspartoacylase in the rat central nervous system. J Comp Neurol. 2004 May 3;472(3):318-29. doi: 10.1002/cne.20080.

4. Gujar SK, Maheshwari S, Björkman-Burtscher I, Sundgren PC. Magnetic resonance spectroscopy. J Neuroophthalmol. 2005 Sep;25(3):217-26. doi: 10.1097/01. wno.0000177307.21081.81.

5. Hoshino H, Kubota M. Canavan disease: clinical features and recent advances in research. Pediatr Int. 2014 Aug;56(4):477-83. doi: 10.1111/ped.12422.

6. Leone P, Shera D, McPhee SW, Francis JS, Kolodny EH, Bilaniuk LT, Wang DJ, Assadi M, Goldfarb O, Goldman HW, Freese A, Young D, During MJ, Samulski RJ, Janson CG. Long-term follow-up after gene therapy for canavan disease. Sci Transl Med. 2012 Dec 19;4(165):165ra163. doi: 10.1126/ scitranslmed.3003454.

7. Janson CG, McPhee SW, Francis J, Shera D, Assadi M, Freese A, Hurh P, Haselgrove J, Wang DJ, Bilaniuk L, Leone P. Natural history of Canavan disease revealed by proton magnetic resonance spectroscopy (1H-MRS) and diffusionweighted MRI. Neuropediatrics. 2006 Aug;37(4):209-21. doi: 10.1055/s-2006-924734.

8. Janson CG, Kolodny EH, Zeng BJ, Raghavan S, Pastores G, Torres P, Assadi M, McPhee S, Goldfarb O, Saslow B, Freese A, Wang DJ, Bilaniuk L, Shera D, Leone P. Mildonset presentation of Canavan's disease associated with novel G212A point mutation in aspartoacylase gene. Ann Neurol. 2006 Feb;59(2):428-31. doi: 10.1002/ana.20787.

9. Mendes MI, Smith DE, Pop A, Lennertz P, Fernandez Ojeda MR, Kanhai WA, et al. Clinically Distinct Phenotypes of Canavan Disease Correlate with Residual Aspartoacylase Enzyme Activity. Hum Mutat. 2017 May;38(5):524-531. doi: 10.1002/humu.23181.

10. Sheikh-Hosseini M, Moarefzadeh M, Alavi-Moghaddam H, Morovvati S. A Novel Mutation in Aicardi-Goutières' Syndrome: A Case Report. Journal of Pediatric Neurology. 2021;19(01):050-3.

11. Arjmand B, Larijani B, Sheikh Hosseini M, Payab M, Gilany K, Goodarzi P, Parhizkar Roudsari P, Amanollahi Baharvand M, Hoseini Mohammadi NS. The Horizon of Gene Therapy in Modern Medicine: Advances and Challenges. Adv Exp Med Biol. 2020;1247:33-64. doi: 10.1007/5584_2019_463.

12. Baslow MH, Guilfoyle DN. Canavan disease, a rare earlyonset human spongiform leukodystrophy: insights into its genesis and possible clinical interventions. Biochimie. 2013 Apr;95(4):946-56. doi: 10.1016/j.biochi.2012.10.023.

13. Hershfield JR, Pattabiraman N, Madhavarao CN, Namboodiri MA. Mutational analysis of aspartoacylase: 
implications for Canavan disease. Brain Res. 2007 May 7;1148:1-14. doi: 10.1016/j.brainres.2007.02.069.

14. Hussain R, Daud S, Kakar N, Ahmad A, Baloch AH, Tareen AM, Kakar MA, Ahmad J. A missense mutation (p.G274R) in gene ASPA causes Canavan disease in a Pakistani family. Mol Biol Rep. 2012 May;39(5):6197-201. doi: 10.1007/s11033011-1438-2.

15. Wijayasinghe YS, Pavlovsky AG, Viola RE. Aspartoacylase catalytic deficiency as the cause of Canavan disease: a structural perspective. Biochemistry. 2014 Aug 5;53(30):4970-8. doi: 10.1021/bi500719k.

16. Zeng BJ, Pastores GM, Leone P, Raghavan S, Wang ZH, Ribeiro LA, Torres P, Ong E, Kolodny EH. Mutation analysis of the aspartoacylase gene in non-Jewish patients with Canavan disease. Adv Exp Med Biol. 2006;576:165-73; discussion 361-3. doi: 10.1007/0-387-30172-0_11.

17. Eke GH, Iscan A, Cece H, Calik M. A mutation of aspartoacylase gene in a Turkish patient with Canavan disease. Genet Couns. 2012;23(1):9-12.

18. Di Pietro V, Cavallari U, Amorini AM, Lazzarino G, Longo S, Poggiani C, Cavalli P, Tavazzi B. New T530C mutation in the aspartoacylase gene caused Canavan disease with no correlation between severity and $\mathrm{N}$-acetylaspartate excretion. Clin Biochem. 2013 Dec;46(18):1902-4. doi: 10.1016/j.clinbiochem.2013.09.004.

19. Bley A, Denecke J, Kohlschütter A, Schön G, Hischke S, Guder P, Bierhals T, Lau H, Hempel M, Eichler FS. The natural history of Canavan disease: 23 new cases and comparison with patients from literature. Orphanet J Rare Dis. 2021 May 19;16(1):227. doi: 10.1186/s13023-020-01659-3.

20. Durmaz AA, Akin H, Onay H, Vahabi A, Ozkinay F. A novel aspartoacylase (ASPA) gene mutation in Canavan disease. Fetal Pediatr Pathol. 2012 Aug;31(4):236-9. doi: 10.3109/15513815.2011.650292.

21. Kaul R, Balamurugan K, Gao GP, Matalon R. Canavan disease: genomic organization and localization of human ASPA to $17 \mathrm{p} 13$-ter and conservation of the ASPA gene during evolution. Genomics. 1994 May 15;21(2):364-70. doi: 10.1006/geno.1994.1278.

22. Kaul R, Gao GP, Aloya M, Balamurugan K, Petrosky A, Michals K, Matalon R. Canavan disease: mutations among Jewish and non-Jewish patients. Am J Hum Genet. 1994 Jul;55(1):34-41.

23. Ashrafi M, Tavasoli A, Katibeh P, Aryani O, VafaeeShahi M. A Novel Mutation in Aspartoacylase Gene; Canavan Disease. Iran J Child Neurol. 2015 Fall;9(4):54-7. 